\title{
Endocarditis infecciosa neonatal: diagnóstico y tratamiento
}

\author{
Pedro Juan Cullen-Benítez, ${ }^{1, *}$ Rocco José González-Morán, ${ }^{1}$ \\ Mónica Magdalena Hidalgo-Vázquez, ${ }^{2}$ Claudia del Carmen López-Enríquez, ${ }^{3}$ \\ Araceli Martínez-Hernández, ${ }^{4}$ Rodrigo Barrón-San Pedro, ${ }^{5}$ José Iglesias-Leboreiro ${ }^{6}$ \\ ${ }^{1}$ Pediatra Neonatólogo; ${ }^{2}$ Residente de Neonatología; ${ }^{3}$ Infectólogo Pediatra; ${ }^{4}$ Residente de Neonatología; \\ ${ }^{5}$ Cardiólogo Pediatra; ${ }^{6}$ Jefe de Departamento de Neonatología. Hospital Español de México, Ciudad de México.
}

\section{RESUMEN}

La endocarditis infecciosa es la inflamación valvular o mural del endocardio causada por microorganismos. En recién nacidos lo más frecuente es que se presente con un cuadro subagudo. Los grupos de riesgo para endocarditis son pacientes con cardiopatías congénitas de alto flujo, postoperados del corazón y quienes han tenido catéteres intravasculares por periodos prolongados, siendo estos últimos los más frecuentes en neonatología. La incidencia anual es de 0.05 a 0.12 por cada 1,000 niños hospitalizados y $7 \%$ en neonatos. Los criterios de Duke y de Li son los más aceptados, que incluyen datos clínicos, de imagen y de microbiología. Los agentes causales más frecuentes son del género Staphylococcus seguidos de los estreptococos; los bacilos Gram negativos y hongos con los enterococos y polimicrobianos son menos frecuentes. El tratamiento consiste en antibióticos por periodos prolongados; al menos uno debe ser bactericida, seleccionando la combinación con base en las resistencias in vitro y adaptado a las resistencias locales conocidas. Deben retirarse los dispositivos centrales al iniciar tratamiento y tomarse cultivos diariamente hasta que sean negativos. El tratamiento quirúrgico se reserva para quienes tienen complicaciones que ponen en peligro la vida.

Palabras clave: Endocarditis infecciosa, recién nacidos, cardiopatía congénita, catéter central, trombo endocárdico, criterios de Duke.

\begin{abstract}
Infectious endocarditis is a valvular or mural endocardial inflammation caused by microorganisms. Subacute presentation is the most frequent form in newborns. Risk factors associated to endocarditis are post-cardiac surgery patients, congenital heart disease, and use of central catheters for prolonged periods of time; the former is the most common in neonates. Annual incidence of endcarditis is 0.05 to 0.12 cases per 1,000 hospitalized children and $7 \%$ in neonates. Duke and Li are the most widely accepted criteria for diagnosis, where clinical evidence, imaging studies and microbiology are mandatory. Staphylococcus followed by Streptococcus are the most frequent causal germs, followed by Gram-negative bacilli, fungus and Enterococcus; polymicrobial germs are the less frequent. Combination of antimicrobial treatment is the cornerstone, where at least one needs to be bactericidal. Antimicrobial combination should be based on in vitro susceptibility and local known epidemiology. Central devices ought to be removed, and blood cultures must be obtained daily until they are become negative. Surgical treatment is reserved for those patients with life threatening complications.
\end{abstract}

Keywords: Infectious endocarditis, newborn, congenital heart disease, central catheter, endocardial thrombus, Duke criteria.

\footnotetext{
* Correspondencia: PJCB, pjuancb@gmail.com

Conflicto de intereses: Los autores declaran que no tienen. Citar como: Cullen-Benítez PJ, González-Morán RJ, HidalgoVázquez MM, López-Enríquez CC, Martínez-Hernández A, Barón-San Pedro $R$ et al. Endocarditis infecciosa neonatal: diagnóstico y tratamiento. Rev Mex Pediatr 2019; 86(5):202-209. [Neonatal infectious endocarditis: diagnosis and treatment]
}

\section{INTRODUCCIÓN}

La endocarditis infecciosa en neonatos es más frecuente que en niños mayores y representa $7 \%$ del total de endocarditis en la edad pediátrica. Está muy relacionada con las cardiopatías congénitas dado que causan flujos turbulentos, pero sobre todo con la presencia prolongada 
de catéteres intravenosos. Este artículo es una revisión sobre el proceso para llevar al diagnóstico basado en el cumplimiento de criterios clínicos, microbiológicos y de imagen; además, se presentan las recomendaciones para el tratamiento.

\section{Definiciones}

La estructura básica del corazón consiste en pericardio, miocardio y endocardio. Este último está compuesto por una capa de endotelio en contacto con el fluido sanguíneo. A partir del endocardio se forman las válvulas cardiacas. ${ }^{1}$ El endotelio tiene un epitelio plano simple con funciones activas en la fisiología cardiovascular: 1) función anti-trombogénica por diversos mecanismos, como la secreción de prostaciclina, factor activador de plasminógeno y degradación de trombina, entre otros. 2) Regulación de lípidos plasmáticos, por el rol en el metabolismo de lipoproteínas de baja y muy baja densidad. 3) Regulación del tono vascular que actúa como intermediario hormonal y sustancias vasoactivas que metabolizan noradrenalina o que transforman angiotensina en angiotensina II. ${ }^{2}$

La endocarditis se define como la inflamación valvular o mural del endocardio por cualquier causa; mientras que la endocarditis infecciosa (EI) es la causada por bacterias u hongos, e involucra el corazón o los grandes vasos (endarteritis) y puede incluir la formación de abscesos. ${ }^{3}$ Los niños con mayor riesgo de desarrollarla son quienes presentan cardiopatía congénita de alto flujo, los sometidos a cirugía cardiaca, quienes requieren la colocación de catéteres intravasculares y pacientes inmunodeprimidos. ${ }^{4}$

\section{Patogenia y fisiopatología}

Se sabe que hay dos condiciones necesarias para la endocarditis: el daño endotelial y los microorganismos que expresan múltiples adhesinas. El daño endotelial ocurre como resultado de flujos turbulentos por estructuras anormales, como prótesis, cardiopatías o por algún dispositivo como catéteres o marcapasos, los cuales pueden causar daño directo por "raspado" de la superficie endotelial o por efecto indirecto debido a flujos turbulentos en la válvula tricúspide. El daño endotelial causa una respuesta de adhesión plaquetaria y depósito de fibrina, lo que produce la formación de un trombo endocárdico no bacterial (TENB) que sirve como nido para el depósito de bacterias u hongos. ${ }^{5}$

$\mathrm{Al}$ existir alguna condición que genere bacteriemia o fungemia transitoria, los gérmenes se alojan en la lesión y dan origen a lo que se conoce como vegetación. La vegetación tiende a crecer por la respuesta inmunológica que se agrega al trombo y fibrina. Las vegetaciones suelen formarse en el lado de baja presión del defecto, que las "protege" del contacto con antibióticos. Las bacterias atrapadas proliferan y hacen crecer la vegetación, liberando constantemente bacterias que, a su vez, producen bacteriemias continuas de bajo grado. ${ }^{4}$

\section{Epidemiología}

La incidencia anual de la EI en Estados Unidos de Norteamérica (EUA) entre 2003 y 2010 se reportó entre 0.05 y 0.12 casos, por cada 1,000 niños hospitalizados. ${ }^{6}$ En niños, durante las últimas dos décadas, las cardiopatías congénitas se han convertido en la enfermedad subyacente principal (hasta 90\%), a diferencia del pasado, cuando la fiebre reumática era la condición que predominaba. En corazones estructuralmente sanos, el uso de líneas de acceso vascular central, en especial en neonatología, constituye un factor de riesgo importante. ${ }^{3}$

La incidencia de esta complicación en los últimos 20 años se ha incrementado, debido al uso de técnicas invasivas para tratar diversos problemas médicos, siendo el principal el uso de catéteres centrales. Por lo anterior, las lesiones en recién nacidos $(\mathrm{RN})$ son más comunes en las estructuras derechas del corazón.

La mortalidad por EI en niños oscila entre 5 y $10 \%$, pero es mayor en pacientes prematuros (13.3\%) que en niños mayores (4.5\%). ${ }^{7}$ En una revisión de 1983 a 1995 de un hospital en Australia, de 12,249 RN atendidos en la unidad neonatal, ocho (0.07\%) tuvieron endocarditis y, la mayoría fue en prematuros menores de 30 semanas de gestación. ${ }^{8}$

Si bien, las cardiopatías congénitas constituyen uno de los factores de riesgo más importantes, por lo general la endocarditis se presenta fuera del periodo neonatal, es decir, durante la infancia o incluso en la vida adulta. ${ }^{9}$ La cirugía cardiaca correctiva de cardiopatías congénitas sin duda reduce el riesgo de EI, especialmente en valvulopatías; sin embargo, la cirugía también puede aumentar el riesgo, sobre todo cuando se realizan cortocircuitos o se colocan prótesis valvulares. ${ }^{10}$

En RN, la prematurez por sí misma es un factor de riesgo, no sólo por su vulnerabilidad a infecciones, sino por la frecuente necesidad de colocar líneas centrales, para nutrición parenteral o administración de medicamentos. ${ }^{11}$ Sin embargo, se ha observado que la formación de TENB puede darse posterior al síndrome de dificultad respiratoria (SDR), en la persistencia de circulación fetal 


\section{Tabla 1: Criterios de Duke y Li (Duke modificado) para diagnóstico de endocarditis infecciosa (El). ${ }^{14}$}

Criterios de Duke
$\begin{aligned} & \text { El definitiva } \\ & -2 \text { criterios mayores, } 0 \\ & -1 \text { mayor y } 3 \text { menores, } 0 \\ & -5 \text { criterios menores }\end{aligned}$

\section{El posible}

No se confirma El definitiva ni se rechaza

\section{El rechazada}

- Diagnóstico alternativo confirmado 0 ,

- Resolución con antibióticos en 4 días 0 menos, 0

- No hay evidencia de endocarditis en cirugía o autopsia
Criterios mayores y menores

\section{Criterios mayores \\ Hemocultivo positivo \\ - Microorganismo típico en 2 hemocul- tivos diferentes: S. viridans, S. bovis, Staphylococcus aureus, Enterococcus, grupo HACEK, 0 \\ - Microorganismo persistente positivo en dos hemocultivos separados de 12 ho- ras, o tres hemocultivos separados de una hora, o un positivo para Coxiella burnetii}

\section{Alteración endocárdica}

- Ecocardiograma positivo: vegetación móvil en válvula, estructura cardiaca, flujo de regurgitación o material implantado, o absceso, o nueva dehiscencia de la válvula prostética

- Nuevo soplo de regurgitación

\section{Criterios menores}

- Condición cardiaca previa o uso de drogas IV

- Fiebre igual o mayor a 38 oC

- Fenómeno vascular

- Fenómeno inmunológico

- Hemocultivo positivo que no satisface criterios mayores

- Ecocardiograma positivo que no satisface criterios mayores

Criterios de Li (Duke modificados)

\section{El definitiva \\ - 2 criterios mayores, 0 \\ - 1 mayor y 3 menores, 0 \\ - 5 criterios menores}

\section{El posible}

- 1 criterio mayor y 1 menor, 0

- 3 criterios menores

\section{El rechazada}

- Diagnóstico alternativo confirmado 0 ,

- Resolución con antibióticos en 4 días 0 menos, 0

- No hay evidencia de endocarditis en cirugía 0 autopsia 0 ,

- No hay criterios para El posible

Adaptado de: Carceller A. Endocarditis infecciosa. An Pediatr (Barc). 2005; 63 (5): 383-389.

o cuando ocurre coagulación intravascular diseminada (CID), ya que todas causan aumento de sustancias vasoactivas que incrementan la agregación plaquetaria. ${ }^{12}$

\section{Diagnóstico}

En general, la presentación clínica de la EI puede ser con síntomas inespecíficos, o bien, con datos de sepsis franca o choque séptico. Los cuatro tipos de presentación clínica de la endocarditis son: 1) cuadro de sepsis por bacteriemia o fungemia, 2) cambios en los ruidos cardiacos por valvulitis o presencia de TENB o vegetaciones, 3 ) lesiones de Roth (raras en niños y excepcionales en neonatos), y 4) embolia renal, abdo- minal, osteomielitis, meningitis o neumonía, con la sintomatología propia de cada problema. ${ }^{13}$

Los RN suelen tener presentación clínica insidiosa con hiporexia, pérdida de peso, dificultad respiratoria o datos francos de choque séptico. Por ello, en esta etapa el diagnóstico de la endocarditis bacteriana requiere de un alto índice de sospecha, tomando en cuenta los datos clínicos y los factores de riesgo. ${ }^{14}$

Desde 1909, en Inglaterra Thomas Horder describió los signos y síntomas de la EI, pero fue hasta 1981 cuando Von Reyn propuso sus criterios para facilitar el diagnóstico, clasificándolo en definitivo, probable, posible y rechazado. ${ }^{15}$ En 1994, en la Universidad de Duke se proponen nuevos criterios 


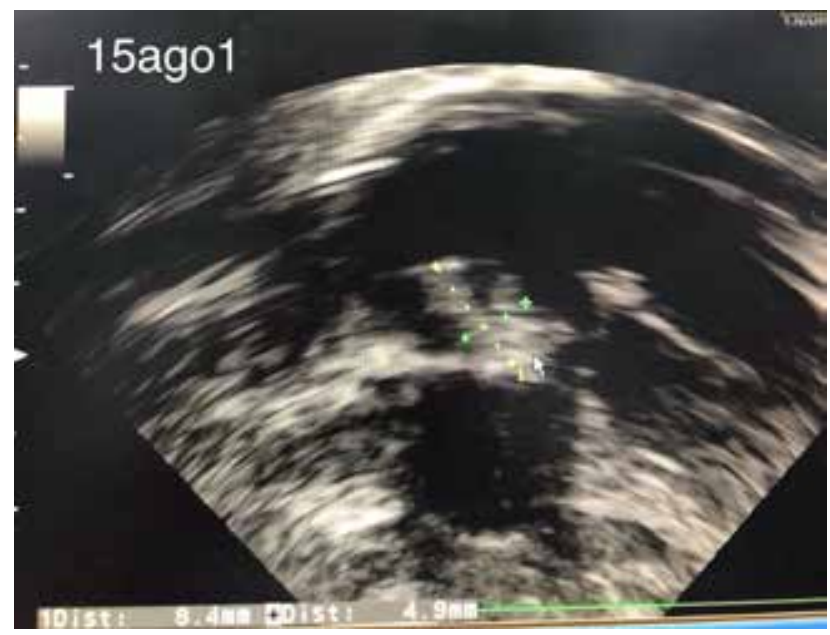

Figura 1: Vegetación de 8.4 × 4.9 mm en válvula tricúspide en neonato de 29 semanas de gestación (35 corregidas).

(criterios de Duke), con tres opciones: endocarditis definitiva, posible o rechazada. ${ }^{16}$ En 2000, Li y colaboradores presentan criterios modificados y más estrictos, conocidos como criterios de Duke modificados, en donde solo se cambió la manera de llegar al diagnóstico posible. ${ }^{17}$

La Tabla 1 detalla las diferencias entre los criterios de Duke y de Li así como los criterios menores y mayores que se usan para ambos. En la columna central de la tabla se muestran los dos criterios mayores y los seis criterios menores, que se usan tanto para Duke como para Li. En los criterios de Duke (descritos en la columna izquierda) para llegar al diagnóstico definitivo de endocarditis se requieren dos criterios mayores, un criterio mayor y dos menores o cinco criterios menores. Se rechaza el diagnóstico si se encuentra un diagnóstico alterno, se resuelve el problema tras cuatro días de tratamiento antibiótico, o bien, cuando no hay evidencia de endocarditis tras cirugía o autopsia. En los casos que no se establece el diagnóstico definitivo, pero tampoco se rechaza, queda como diagnóstico posible. En cambio, en los criterios de $\mathrm{Li}$ el diagnóstico definitivo se hace con los mismos requisitos que en los de Duke, pero se considera diagnóstico posible si tiene un criterio mayor y uno menor, o tres menores. Se rechaza el diagnóstico si no cumple con los criterios de diagnóstico definitivo ni posible, o bien, al cumplir con la definición de rechazo con los criterios de Duke. ${ }^{14}$ Los criterios de Duke tienen una sensibilidad de $80 \%$ y, aunque se describieron para adultos, siguen siendo un punto de referencia aun en niños y neonatos.
El diagnóstico se basa en datos clínicos, de imagen y microbiológicos. ${ }^{18}$ En todas las edades, el ecocardiograma es el estándar de oro para la identificación de la vegetación; en la mayoría, la ecocardiografía transtorácica es suficiente, pero a veces la transesofágica puede ser de utilidad. La Figura 1 muestra el caso de una vegetación de 8.4 x 4.9 mm de nuestra institución en el que se cumplieron los dos criterios mayores para hacer diagnóstico definitivo. Recientemente se ha descrito la utilidad de la tomografía por emisión de positrones, en particular en las endocarditis relacionadas a cardiopatías congénitas. ${ }^{19}$

La documentación microbiológica se realiza por hemocultivo. Si bien las nuevas técnicas de reacción en cadena de polimerasa (PCR) han avanzado, permitiendo mayor rapidez para la identificación del agente causal y de algunas especies resistentes, como mecA de Staphylococcus aureus, estas técnicas aún no sustituyen el hemocultivo. ${ }^{20}$

Los hemocultivos deben ser periféricos y no de catéteres; la toma requiere de al menos tres cultivos en 24 a 48 horas. Cabe señalar que hasta en 10\% de los casos no se obtienen hemocultivos positivos. ${ }^{21}$ Con respecto a la toma de hemocultivos, las recomendaciones (incluyendo pacientes pediátricos) más aceptadas son:

a) Tomar hemocultivos en todo paciente con fiebre y datos de sepsis sin origen claro y con soplo; o bien, pacientes con historia de cardiopatía o endocarditis previa.

b) Deben tomarse al menos tres cultivos, de diferentes punciones, en el primer día y si no hay crecimiento en el primer día, obtener dos o más de tres hemocultivos.

c) En pacientes que no parecen enfermos y los cultivos permanecen negativos por más de 48 horas, es razonable retrasar el inicio de antibióticos y obtener nuevos cultivos.

d) En pacientes severamente enfermos, se deben obtener tres hemocultivos con separación de una hora e iniciar tratamiento empírico inmediato.

e) No es recomendable tomar hemocultivos arteriales.

En RN, especialmente en prematuros de bajo peso, el volumen de extracción puede limitarse a sólo $1 \mathrm{~mL}$ y el número de cultivos puede ser menor de tres, en las primeras 48 horas. $^{5}$

\section{Agentes causales}

En todas las edades, los géneros Staphylococcus y Streptococcus son responsables de $55-95 \%$ de las EI 
de válvulas nativas. La Tabla 2 muestra también que los Streptococcus son menos comunes en los neonatos que en el resto de la edad pediátrica, mientras que los Staphylococcus aureus y coagulasa negativo tienen más frecuencia en la edad neonatal. Los bacilos Gram negativos y los hongos también son más frecuentes en $\mathrm{RN}$ que en niños mayores de dos meses. Enterococcus se presentan en menos de $1 \%$ de las endocarditis neonatales y hasta en $6 \%$ del resto de los niños.

Los bacilos conocidos como grupo HACEK (Haemophilus parainfluenzae, $H$. aphrophilus, $H$. paraphrophilus, $H$. influenzae, Actinobacillus actinomycetemcomitans, Cardiobacterium hominis, Eikenella corrodens, Kingella kingae, $K$. denitrificans), así como aquéllos con hemocultivo negativo, se presentan en 2 a $6 \%$ de los neonatos y, de 0 a $16 \%$ de los niños. Por último, los hemocultivos polimicrobianos suceden en 3 a $5 \%$ en $\mathrm{RN}$, pero en menos de $1 \%$ de los niños. ${ }^{22}$

La virulencia de algunas especies como estafilococos, enterococos y estreptococos es mayor debido a las adhesinas que son clave para el desarrollo de la endocarditis. Las adhesinas del Staphylococcus aureus han sido las más estudiadas, referidas como MSCRAMM(Microbial Surface Components Recognizing Adhesive Matrix Molecules). Se fijan a colágeno, laminina, fibrinógeno y fibronectina, componentes presentes en el endocardio lesionado. ${ }^{5}$

La endocarditis fúngica es relevante, pues representa de 8 a 10\% del total. En una revisión sistemática, publicada en el 2017, se buscaron todas las referencias sobre endocarditis por hongos en niños. Se encontró que, de 93 casos reportados en menores de un año, 60 fueron neonatos (64\%) y de ellos, 57 fueron prematuros (95\%) con una media de edad gestacional al nacer de
27 semanas y peso de $860 \mathrm{~g}$ promedio. Los factores de riesgo más importantes fueron la prematurez, aplicación de catéteres centrales, uso previo de antibióticos y la cirugía de corazón abierto. El sitio de infección más frecuente fue el corazón derecho. El pronóstico de la endocarditis fúngica sigue siendo malo; en esta serie la tasa de fatalidad fue de $56 \%$, aunque esto ha mejorado después del año 2000 cuando se reporta tasa de mortalidad de $33 \%$. Candida albicans (60\%) es lo más frecuente en $\mathrm{RN}$, seguida de C. parapsilosis (12\%), Candida spp. (10\%) y C. tropicalis (5\%). ${ }^{23} \mathrm{Al}-$ gunas características biológicas únicas de la población pediátrica, pero especialmente la neonatal, ayudan al desarrollo de fungemias: los menores diámetros vasculares que obligan al uso de catéteres de poco calibre proveen un nido para tromboflebitis y endocarditis. Además, la inmadurez inmunológica monocítica y de la fagocitosis polimorfonuclear los pone en situación especial de vulnerabilidad. ${ }^{24}$

\section{Tratamiento}

La EI es un problema complejo y requiere la intervención de varias especialidades como infectología, cardiología y cirugía cardiovascular. El tratamiento consiste principalmente en antibióticos por tiempo prolongado, pero se puede requerir cirugía en casos de falla cardiaca, infección fuera de control o prevención de embolismo inminente. Aún quedan muchas áreas de incertidumbre sobre el tratamiento de la EI, entre las cuales está la correcta duración del tratamiento, el uso de tratamiento oral, el momento apropiado para iniciarlo y el momento óptimo de realizar la cirugía. ${ }^{18}$

\section{Tabla 2: Frecuencia de agentes causales en diferentes edades para válvulas nativas. ${ }^{22}$}

\begin{tabular}{lccc} 
Microorganismo & Neonatos $\%$ & 2 meses-15 años $\%$ & $16-60$ años \% \\
\hline Streptococcus spp. & $15-20$ & $40-50$ & $45-65$ \\
Staphylococcus aureus & $40-50$ & $22-27$ & $30-40$ \\
Staphylococcus coagulasa negativo & $8-12$ & $4-7$ & $4-8$ \\
Enterococcus spp. & $<1$ & $3-6$ & $5-8$ \\
Bacilos Gram negativos & $8-12$ & $4-6$ & $4-10$ \\
Hongos & $8-12$ & $1-3$ & $1-3$ \\
Cultivos negativos y organismos HACEK & $2-6$ & $0-15$ & $3-10$ \\
Difteroides & $<1$ & $<1$ & $<1$ \\
Polimicrobianos & $3-5$ & $<1$ & $1-2$ \\
\hline
\end{tabular}

Adaptado de: Mylonakis E, Calderwood SB. Infective endocarditis in adults. N Eng J Med. 2001; 345 (18): 1318-1330. 
Tabla 3: Diferencias en las recomendaciones de 2015 de la AHA y la ASC para el tratamiento de la endocarditis infecciosa (EI) en niños.

\section{American Heart Association (AHA) \\ Tratamiento de Streptococcus spp.}

No se recomienda tratamiento corto (2 semanas) en El no complicada

MIC para alta susceptibilidad a penicilina $<0.1 \mathrm{mg} / \mathrm{L}$

MIC para cepa relativamente resistente $>0.2 \mathrm{mg} / \mathrm{L}$

Tratamiento de Staphylococcus spp.

El de válvula nativa: oxacilina en cepas susceptibles a meticilina; se puede usar gentamicina en los primeros 3 a 5 días

No se menciona la necesidad del retraso de la rifampicina en prótesis valvulares

La daptomicina se recomienda como agente alterno para pacien-

tes con alergia a betalactámicos y como alternativa a vancomicina

\section{Tratamiento de Enterococcus spp.}

Ampicilina y ceftriaxona se recomienda en cepas con resistencia 0 intolerancia a aminoglucósidos durante todo el tratamiento

\section{European Society of Cardiology (ASC)}

El tratamiento corto (2 semanas) en El no complicada no se descarta

MIC para alta susceptibilidad a penicilina $<0.0125 \mathrm{mg} / \mathrm{L}$

MIC para cepa relativamente resistente de 0.250 a $2.0 \mathrm{mg} / \mathrm{L}$

El de válvula nativa con oxacilina en cepas susceptibles a meticilina; gentamicina no se recomienda por otoxicidad y nefrotoxicidad

Con prótesis valvular, la adición de rifampicina puede retrasarse de 3

a 5 días de terapia efectiva con oxacilina o vancomicina

La daptomicina se recomienda como agente alterno para pacientes con alergia a betalactámicos y como alternativa a vancomicina, dado que es superior a vancomicina para Staphylococcus aureus susceptible y resistente a meticilina con MIC a vancomicina $>0.1 \mathrm{mg} / \mathrm{L}$

Ampicilina y ceftriaxona para El por $E$. faecalis puede usarse con o sin resistencia a aminoglucósidos. En cepas susceptibles, algunos expertos recomiendan dos semanas de gentamicina una vez al día

\section{Terapia antimicrobiana}

El tratamiento de la EI consiste en antibióticos por periodos prolongados, debido a que las bacterias alojadas en el trombo endocárdico no reciben flujo sanguíneo directo, por lo que el acceso del medicamento es limitado. Además algunos microorganismos en esas circunstancias forman un biofilm que les confiere cierta protección incluso contra antibióticos. Existen cinco principios básicos para el tratamiento antibiótico de la EI: $5,20,25$

1) De los medicamentos empleados, al menos uno debe ser bactericida.

2) El tratamiento es necesariamente prolongado, de cuatro a ocho semanas.

3) Debe privilegiarse la vía intravenosa sobre la intramuscular.

4) Tanto el tratamiento empírico como el dirigido deben adaptarse a las necesidades de resistencia de la unidad médica local, usando las guías internacionales.

5) Al completar el tratamiento, es innecesario tomar hemocultivos cuando no hay síntomas sugestivos. Al iniciar tratamiento, deben tomarse hemocultivos todos los días hasta que sean negativos, para documentar el momento en que cesó la bacteriemia.
Las recomendaciones para el tratamiento publicadas por la Asociación Americana del corazón (AHA) y por la Sociedad Europea de Cardiología (ESC) son muy similares, pero varían en algunos detalles. La Tabla 3 muestra dichas diferencias. 5,26

La bacteriemia por Staphylococcus aureus a menudo es causada por dispositivos centrales, por lo que los catéteres deben removerse para acelerar la resolución, ya que de lo contrario la bacteriemia puede perdurar, aumentando el riesgo de siembras extracardiacas. ${ }^{25}$ Las infecciones por Staphylococcus aureus resistente a meticilina (MSRA), comunes en algunas unidades hospitalarias y de transmisión habitual por el personal de salud, requieren tratamiento con vancomicina y rifampicina debido a que la primera no es bactericida; se recomienda daptomicina como alternativa a rifampicina. ${ }^{27}$

La EI por enterococos tiene tasas de mortalidad elevadas. Enterococcus faecalis y E. faecium son las especies más relevantes, aunque $97 \%$ son por $E$. faecalis y es menos frecuente su resistencia antibiótica. Sin embargo, su habilidad para formar biofilm la hace de difícil tratamiento al ocasionar resistencia a aminoglucósidos en más de $50 \%$ de las ocasiones. ${ }^{28,29}$ En 2015 las guías europeas para EI recomiendan el esquema de ampicilina y cefotaxima contra $E$. faecalis resistente y no resistente a aminoglucósidos en clase IIa y nivel B de evidencia. ${ }^{5}$ 
En las endocarditis causadas por el grupo de bacterias HACEK se recomienda un curso de cuatro semanas de ceftriaxona con otra cefalosporina de tercera generación, o bien, ampicilina y gentamicina. En los casos de otros microorganismos Gram negativos como Pseudomonas sp. Escherichia coli o Serratia $s p$. se recomienda una combinación de penicilina de espectro extendido (piperacilina/tazobactam), o bien, cefalosporina (ceftazidima o ceftriaxona) más aminoglucósido, por un mínimo de seis semanas. ${ }^{5}$

Las endocarditis con cultivo negativo son un problema serio porque el tratamiento empírico empleado puede no cubrir la sensibilidad del germen y porque el mismo tratamiento puede causar efectos adversos. Por esta razón, el comité de la AHA recomienda no iniciar antibióticos hasta tener un cultivo positivo o suspender cualquier antibiótico que se haya iniciado en los cuatro días previos. El tratamiento empírico debe basarse en el evento pivote que probablemente haya desencadenado el evento, así como la exposición previa a antibióticos, si la infección es adquirida en comunidad o nosocomial, el tipo de válvula afectada (nativa o de prótesis), y el tipo de infección (aguda o subaguda). El consenso de los expertos es que el régimen inicial sea con ampicilina/sulbactam más gentamicina por cuatro a seis semanas, o vancomicina más gentamicina igualmente por cuatro a seis semanas y ciprofloxacina para válvulas nativas. En el caso de prótesis valvulares, se debe considerar vancomicina por seis semanas, adicionando gentamicina por dos semanas más cefepime por seis semanas, más rifampicina por seis semanas. ${ }^{5}$
Las endocarditis fúngicas se tratan típicamente con anfotericina $\mathrm{B}$, a pesar de su mala penetración en la vegetación y por la posibilidad de afectar la función renal. Para pacientes con daño renal se recomienda la forma liposomal de anfotericina; las equinocandinas (caspofungina) tienen menos nefrotoxicidad y teóricamente mejores resultados en los biofilms de Candida, pero los resultados de su uso in vivo son contradictorios y aún faltan estudios comparativos. ${ }^{23}$ El fluconazol oral y otros antimicóticos se han usado como tratamiento profiláctico postendocarditis por seis a 12 meses e incluso usándola de manera sinérgica con anfotericina. En una revisión de Pana y colaboradores se reporta que la combinación de antifúngicos se asocia a menos mortalidad que la monoterapia. ${ }^{30}$

\section{Cirugía}

La intervención quirúrgica de la EI puede salvar la vida del paciente y si bien hay indicaciones (Tabla 4), la decisión debe ser individual. Las indicaciones comunes incluyen insuficiencia cardiaca, obstrucción valvular, extensión perivalvular de la infección, endocarditis fúngica, bacteriemia persistente, prótesis inestable, ruptura del seno de Valsalva o septum y eventos embólicos. La cirugía no debe retrasarse por no haber terminado el curso de antibiótico o por continuar con bacteriemias. Algunos pacientes con absceso miocárdico o extensión perianular de la infección pueden no requerir cirugía, especialmente ante la ausencia de bloqueo cardiaco, dehiscencia valvular o insuficiencia cardiaca. ${ }^{31}$

\section{Tabla 4: Indicaciones para cirugía por endocarditis.}

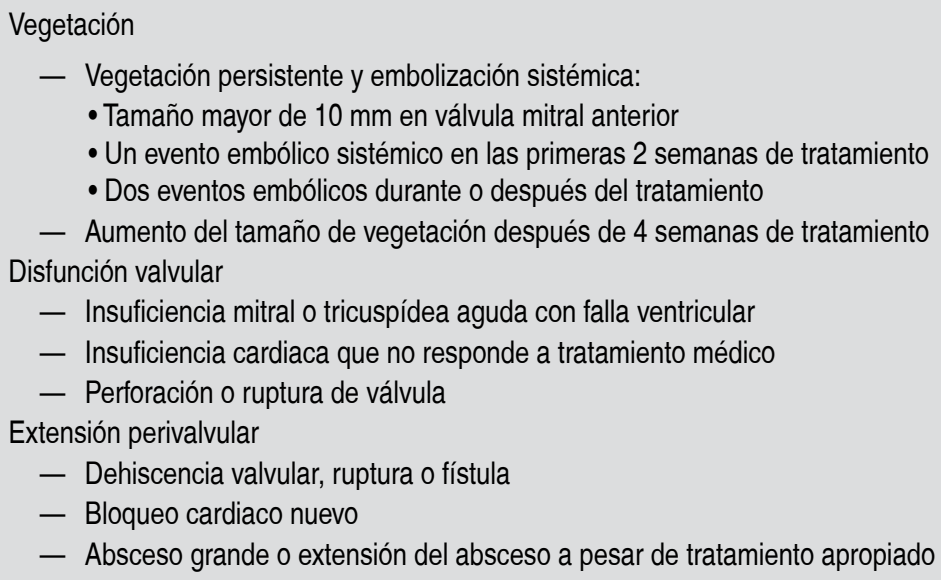




\section{Otro manejo}

La terapia trombolítica suele considerarse cuando hay indicaciones quirúrgicas. Existen la uroquinasa, la estreptoquinasa y el activador de plasminógeno tisular recombinante (rTPA), pero los dos primeros no son específicos a fibrina y son altamente antigénicos, por lo que están contraindicados en neonatos. El rTPA cataliza la conversión de plasminógeno en plasmina con una vida media muy corta, dando una fibrinólisis sin proteinolisis. No debe iniciarse si el paciente ha tenido crisis convulsivas en las últimas 48 horas, sangrado activo, deficiencias de coagulación, procedimientos invasivos en los últimos tres días, trombocitopenia, niveles bajos de fibrinógeno, hipertensión o sepsis severa. La dosis habitual es de 0.1 a $0.6 \mathrm{mg} / \mathrm{kg} / \mathrm{hora}$ por seis a 12 horas por tres días. ${ }^{32}$

\section{REFERENCIAS}

1. Welsch U. Aparato cardiovascular. En: Welsch U. Histología. 2a edición. Madrid, España: Editorial Panamericana; 2010. pp. 235-258.

2. Aleixandre de Artiñano MA, Ortega-Mateo Al. Importancia fisiológica del endotelio. En: Aleixandre de Artiñano MA, OrtegaMateo Al. Función y disfunción endotelial. Madrid, España: Editorial Complutense; 2000. pp. 1-15.

3. Hoyer A, Silberbach M. Infective endocarditis. Pediatr Rev. 2005; 26 (11): 394-400.

4. Olivera AC, Libian LS. Endocarditis infecciosa. Tratamiento y profilaxis. En: Zabala AJI. Protocolos diagnósticos y terapéuticos en cardiología pediátrica. 2010. Sociedad Española de Cardiología Pediátrica y Cardiopatías Congénitas. Disponible en: www.secardioped.org/readcontents.php?file=webstructure/ protocolos_2010.pdf

5. Baltimore RS, Gewitz M, Baddour LM, Beerman LB, Jackson MA, Lockhart PB et al. Infective endocarditis in childhood: 2015 update: a scientific statement from the American Heart Association. Circulation. 2015; 132 (15): 1487-1515.

6. Pasquali SK, He X, Mohamad Z, McCrindle BW, Newburger JW, $\mathrm{Li}$ JS et al. Trends in endocarditis hospitalizations at US children's hospitals: impact of the 2007 American Heart Association antibiotic prophylaxis guidelines. Am Heart J. 2012; 143: 894-899.

7. Day MD, Gauvreau K, Schulman S, Newburger JW. Characteristics of children hospitalized with infective endocarditis. Circulation. 2009; 119 (6): 865-870.

8. Opie FG, Fraser SH, Drew JH, Drew S. Bacterial endocarditis in neonatal intensive care. J Pediatr Child Health. 1999; 35: 545-548.

9. Knirsch W, Nadal D. Infective endocarditis in congenital heart disease. Eur J Pediatr. 2011; 170 (9): 1111-1127.

10. Taib R. Infective endocarditis in children. Southeast Asian J Trop Med Public Health. 2014; 45 Suppl 1: 79-85.

11. Mecrow IK, Ladusans EJ. Infective endocarditis in newborn infants with structurally normal hearts. Acta Paediatr. 1994; 83 (1): 35-39.

12. Walsh MJ, Avinash KS. Infective endocarditis. En: Ungerleider RM, Meliones JN, McMillan KN, Cooper DS, Jacobs JP. Critical heart disease in infants and children. 3rd ed. Philadelphia PA: Elsevier; 2019. pp. 823-835.
13. Fye WB, Fernel JF. Profiles in cardiology. Clin Cardiol. 1997; 20: 1037-1038.

14. Carceller A. Endocarditis infecciosa. An Pediatr (Barc). 2005; 63 (5): 383-389.

15. Von Reyn CF, Levy B, Arbeit RD, Friedland G, Crumpacker C. Infective endocarditis: an analysis based on strict case definitions. Ann Intern Med. 1981; 94: 505-518.

16. Durack DT, Lukes AS, Bright DK. New criteria for diagnosis of infective endocarditis: utilization of specific echocardiographic findings. Duke Endocarditis Service. Am J Med. 1994; 96 (3): 200-209.

17. Li JS, Sexton DJ, Mick N, Nettles R, Fowler VG, Ryan T et al. Proposed modifications to the Duke criteria for the diagnosis of infective endocarditis. Clin Infect Dis. 2000; 3 (4): 633-638.

18. Hoen B, Duval X. Infective endocarditis. N Eng J Med. 2013; 368: 1425-1433.

19. Meyer Z, Fischer M, Koerfer J, Laser KT, Kececioglu D, Burchert $W$ et al. The role of FDG-PET-CT in pediatric cardiac patients and patients with congenital heart defects. Int J Cardiol. 2016; 220: 656-660.

20. Dixon G, Christov G. Infective endocarditis in children. Curr Opin Infent Dis. 2017; 30: 257-267.

21. Stockheim JA. Cardiac infections. En: Rudolph CD. Rudolpfh's pediatrics. 22nd edition. New York: McGraw Hill; 2011.

22. Mylonakis $\mathrm{E}$, Calderwood SB. Infective endocarditis in adults. $N$ Eng J Med. 2001; 345 (18): 1318-1330.

23. Ganesan V, Ponnusamy SS, Sundaramurthy R. Fungal endocarditis in paediatrics: a review of 192 cases (1971-2016). Cardiol Young. 2017; 27 (8): 1481-1487.

24. Andreas HG, Emmanuel R, Thomas JW. Chapter 22, Section 3: Fungal infections in pediatric patients. En: Clinical mycology. Thu Nguyen/Sue Hodgson, 481-500, Elsevier Health Sciences, UK; 2009.

25. Santoro J, Ingerman M. Response to therapy: relapses and reinfections. En: Kaye D, ed. Infective endocarditis. 2nd ed. New York, NY: Raven Press; 1992. pp. 423-434.

26. Habib G, Lancellotti P, Antunes MJ, Bongiomi MG, Casalta JP, Del Zotti $F$ et al. 2015 ESC Guidelines for the management of infective endocarditis: the Task Force for the Management of Infective Endocarditis of the European Society of Cardiology (ESC). Endorsed by: European Association for Cardio-Thoracic Surgery (EACTS), the European Association of Nuclear Medicine (EANM). Eur Heart J. 2015; 36: 3075-3128.

27. Nelson MU, Gallagher PG. Methicillin-resistant Staphylococcus aureus in the neonatal intensive care unit. Semin Perinatol. 2012; 36 (6): 424-430.

28. Beganovic M, Luther MK, Rice LB, Arias CA, Rybak MJ, LaPlante $\mathrm{KL}$. A review of combination antimicrobial therapy for Enterococcus faecalis bloodstream infections and infective endocarditis. Clin Infect Dis. 2018; 67 (2): 303-309.

29. Fernández-Hidalgo N, Almirante B, Gavaldà J, Gurgui M, Peña $\mathrm{C}$, de Alarcón A et al. Ampicillin plus ceftriaxone is as effective as ampicillin plus gentamicin for treating enterococcus faecalis infective endocarditis. Clin Infect Dis. 2013; 56 (9): 1261-1268.

30. Pana ZD, Dotis J, losifidis E, Roilides E. Fungal endocarditis in neonates: a review of seventy-one cases (1971-2013). Pediatr Infect Dis J. 2015; 34 (8): 803-808.

31. Ferrieri P, Gewitz MH, Gerber MA, Newburger JW, Dajani AS, Shulman ST et al. Unique features of infective endocarditis in childhood. Pediatrics. 2002; 109 (5): 931-943.

32. Ozdemir AA. Recombinant tissue plasminogen activator treatment for intracardiac vegetation in a very low birth weight infant. Arch Argent Pediatr. 2017; 115 (5): e307-e310. 